\title{
Reversible Switching of Ultrastrong Light-Molecule Coupling
}

\author{
T. Schwartz, J. A. Hutchison, C. Genet, and T. W. Ebbesen* \\ ISIS, Université de Strasbourg and CNRS (UMR 7006), 8 allée Gaspard Monge, 67000, Strasbourg, France
}

(Received 1 February 2011; revised manuscript received 29 March 2011; published 11 May 2011)

\begin{abstract}
We demonstrate that photochromic molecules enable switching from the weak- to ultrastrong-coupling regime reversibly, by using all-optical control. This switch is achieved by photochemically inducing conformational changes in the molecule. Remarkably, a Rabi splitting of $700 \mathrm{meV}$ is measured at room temperature, corresponding to $32 \%$ of the molecular transition energy. A similar coupling strength is demonstrated in a plasmonic structure. Such systems present a unique combination of coupling strength and functional capacities.
\end{abstract}

DOI: 10.1103/PhysRevLett.106.196405

PACS numbers: $71.36 .+\mathrm{c}, 42.70 . \mathrm{Gi}$

The interaction of light with matter changes profoundly when it occurs in a photonic structure. A classical example is enhancement of fluorescence in a cavity, known as the Purcell effect [1]. Such modifications occur because the emitter and the cavity mode interact and exchange energy, modifying possible emission channels. In the strongcoupling (SC) regime, this exchange becomes faster than any dissipation or decoherence, and the energy bounces back and forth, in what is known as vacuum Rabi oscillations [2-5]. This effect is manifested as a splitting of the cavity spectral response and the formation of two energy branches in the cavity dispersion, and in this situation the system must be viewed as a single entity. At resonance, the energy gap between the two branches, known as Rabi splitting, is given by $\hbar \Omega_{R}=\sqrt{4 V^{2}-\left(\Gamma_{c}-\Gamma_{e}\right)^{2}}$, with $V$ the coupling parameter, $\Gamma_{c}$ the cavity linewidth, and $\Gamma_{e}$ the nonradiative linewidth of the emitter [4].

Originally studied with atoms [2,3], cavity SC was later demonstrated with inorganic semiconductors [4-9]. These were found to have many fascinating applications, such as optical amplification [8], polariton condensation and lasing [9] and quantum information processing [10]. Following the observation of SC in a cavity filled with cyanine $J$ aggregates [11], there has been increasing interest in lightexciton SC using organic molecules [12-16]. Dye molecules have unusually strong transition dipoles, and, since $V$ is proportional to the dipole-matrix element of the transition, SC between a molecular transition and a cavity mode can reach Rabi-splitting values more than 10 times higher than in inorganic semiconductor cavities $[12,13]$.

In fact, one should notice that in these experiments the Rabi frequency is a substantial fraction $(\sim 15 \%)$ of the molecular transition energy, suggesting that such systems could potentially go into the regime of ultrastrong coupling. Ultrastrong coupling is an emerging field in light-matter interaction [17-19], where deviations from the Jaynes-Cummings model appear as the coupling energy approaches that of the coupled transitions [17]. In this regime, the above expression for the Rabi splitting does not hold anymore, and new processes were predicted to give rise to intriguing phenomena such as a squeezed vacuum and generation of correlated photons [17]. To date, ultrastrong coupling was achieved by using intersubband transitions in semiconductor structures in terahertz frequencies or superconducting circuits in the gigahertz regime $[18,19]$. In that context, organic molecules naturally lend themselves to the observation of ultrastrong coupling in the visible spectrum.

Here we demonstrate switchable light-molecule interaction in a cavity containing photochromic spiropyran molecules [20], reaching a Rabi splitting as high as $700 \mathrm{meV}$, which is $32 \%$ of the molecular transition energy. This process is reversible and optically addressable which allows a fine control of the coupling conditions. We also demonstrate similar coupling strength between surface plasmons and the photochrome.

Our system is a low- $Q$ metallic cavity [13] filled with photochromic spiropyran (SPI) molecules $\left(1^{\prime}, 3^{\prime}\right.$-dihydro- $1^{\prime}$, $3^{\prime}, 3^{\prime}$-trimethyl-6-nitrospiro[2H-1-benzopyran-2, $2^{\prime}-(2 H)$ indole]) in a PMMA (polymethyl methacrylate) matrix. Such molecules can undergo photoisomerization between a transparent SPI form and a colored merocyanine (MC) form [20] and were used for optical switching [21]. We begin by sputtering a $35 \mathrm{~nm}$ thick Ag layer on a quartz substrate. Then a PMMA-toluene solution $(3.3 \mathrm{wt} \%$ PMMA, molar weight 120000 ) is prepared with SPI to give a weight ratio of 3:2 SPI to PMMA and spin-cast on the $\mathrm{Ag}$ film at $1850 \mathrm{rpm}$, adjusted to tune the empty-cavity resonance (see below). The cavity is formed by sputtering another $35 \mathrm{~nm}$ layer of $\mathrm{Ag}$.

The spectral evolution of a bare molecular film is shown in Fig. 1(a). The molecules are initially in the SPI state, which is transparent across the visible range and absorbs in the UV [Fig. 1(a), dashed line]. Upon UV irradiation at $334 \mathrm{~nm}$, the molecules are photoisomerized into the MC state, with an absorption peak at $560 \mathrm{~nm}$. This absorbance gradually increases as molecules are converted, until a photostationary state is reached (solid line). In order to explore this phenomenon in the SC regime, a cavity was prepared and characterized by its transmission spectrum, 


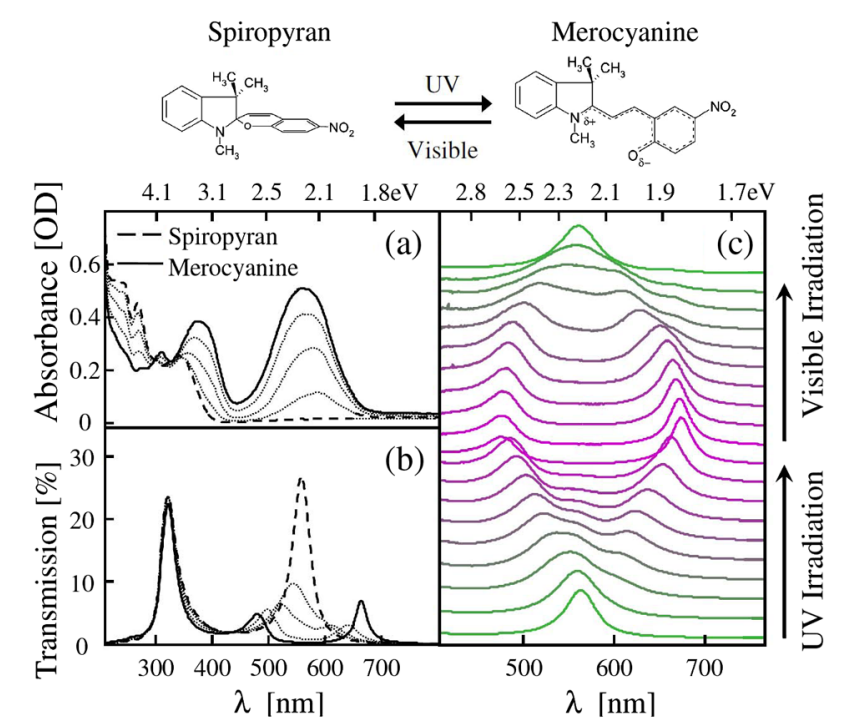

FIG. 1 (color online). (a) Absorption spectra for a neat film in the SPI (dashed line) and MC (solid line) forms. (b) Cavity normal-incidence transmission with the molecules in the SPI (dashed line) and MC (solid line) forms. The dotted lines in (a) and (b) were taken at a few intermediate states. (c) All-optical reversible switching of SC, showing the evolution of the transmission spectrum during conversion. The time scale for photoconversion is $\sim 10 \mathrm{~min}$ at $\sim 1 \mathrm{~mW} / \mathrm{cm}^{2}$, compared to 1 day for thermal backconversion at room temperature.

shown in Fig. 1(b). With the SPI isomer, the system starts totally uncoupled and the cavity exhibits a single resonance with a $Q$ factor of $\sim 10$, centered around $560 \mathrm{~nm}$ [dashed line, Fig. 1(b)]. As molecules are converted into the MC state, the cavity mode gradually broadens due to absorption. The system is weakly coupled until the MC concentration is sufficient to reach the conditions for strong coupling. The transmission spectrum splits symmetrically into two well-defined peaks [Fig. 1(b)] corresponding to the two hybrid modes, reaching an energy difference of $713 \mathrm{meV}$ between them at the photostationary state. This Rabi splitting is $1 / 3$ of the transition energy of the molecular resonance $(2.2 \mathrm{eV})$, placing our system well into the ultrastrong-coupling regime. The system can be reversed through visible-light irradiation [Fig. 1(c)]. The Rabi splitting decreases with irradiation time until the peaks merge and the original single cavity mode is reestablished. The all-optical tunability of the coupling strength is an ideal tool to study how the system properties change as it crosses from weak to ultrastrong coupling, in a clean and simple way, since all other parameters remain fixed. In that sense, it is analogous to early experiments where the number of atoms occupying the cavity was varied [2]. Similar control was recently demonstrated in the terahertz regime with semiconductors, where the charge carrier density was controlled by external voltage [22] or a laser pulse for ultrastrong coupling [19]. We emphasize, however, that here we achieve such a control in a simple configuration using molecular functionality.

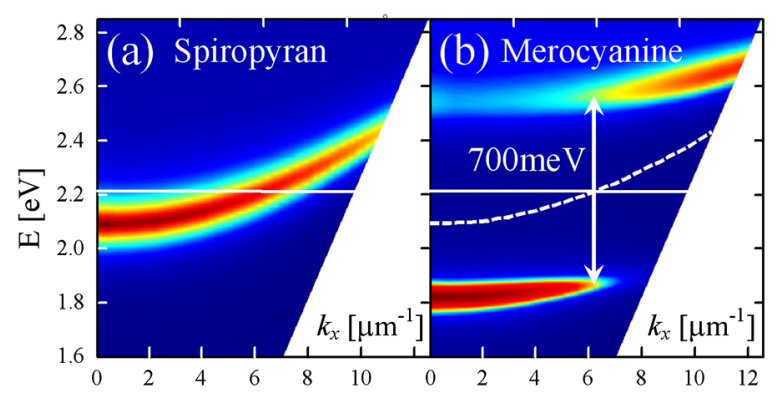

FIG. 2 (color online). Cavity dispersion, measured by angledependent transmission (TE polarization, $0-60^{\circ}$ ) in the SPI (a) and $\mathrm{MC}$ (b) forms, showing the splitting into the upper and lower polariton branches. The solid line marks the molecular transition, and the dashed line is the SPI-state dispersion of (a).

Next, we measure the cavity dispersion in the two forms by acquiring the angle-dependent transmission spectrum. In order to scan the dispersion at both sides around the resonant-coupling point, we fabricate a slightly thicker cavity (by spin coating at $1600 \mathrm{rpm}$ ), resulting in a normal-incidence transmission peak at $594 \mathrm{~nm}$ for the empty cavity. When the molecules are in the SPI form, the cavity energy diagram has a single branch [Fig. 2(a)], and the resonance energy increases with incidence angle. In the MC form [Fig. 2(b)], the dispersion splits into the upper and lower polariton branches of the strongly coupled system. At the resonant-coupling point, namely, when the two original curves cross each other, the energy difference between the two branches is $700 \mathrm{meV}$. In terms of time scales, the Rabi period is $6 \mathrm{fs}$, compared with a cavity lifetime of $25 \mathrm{fs}$ and an excited state lifetime $\sim 50 \mathrm{ps}$ for the molecule. This verifies that our system is still dominated by coherent interactions, despite the relatively high dissipation rates.

In these experiments, the Rabi splitting is measured in transmission; however, the splitting value depends on the type of measurement-transmission, reflection, absorption, or emission [4]. In order to ascertain the nature of the coupling, we analyze the system numerically and compare the results with experiments. As our system operates in the semiclassical (many-molecules) regime, its optical response can be modeled by inserting the molecular contribution to the refractive index into Maxwell's equations [23]. We utilize the transfer matrix formalism and use the measured absorption spectrum of the molecule (in its two forms) with Kramers-Krönig relations to calculate the complex refractive index. The cavity length is found to be $126 \mathrm{~nm}$ by fitting the SPI-form cavity mode (see Fig. 1), and the PMMA is taken with a refractive index of 1.49. Figure 3(a) shows the field amplitude distribution $|E|$ inside and outside the cavity (omitting the incoming radiation, taken with a constant amplitude of 1) as a function of wavelength, with the molecules in the SPI form. Thus, the field emanating below the bottom $\mathrm{Ag}$ layer is the transmission amplitude $\tau$ with its spectrum $\tau^{2}$ plotted below (solid line), while the field above the top Ag layer 
is the reflection amplitude $\rho$. The empty-cavity mode at $560 \mathrm{~nm}$ exhibits a field enhancement of about 3 times relative to the incoming field, matching a $Q$ factor of order 10. In addition, we plot the absorption spectrum $A=1-$ $\tau^{2}-\rho^{2}$ [dashed-dotted line in Fig. 3(a)]. We repeat these calculations for the MC form, with the results in Fig. 3(b). Here one finds that the original cavity resonance has been replaced with the two modes associated with SC, separated by $\Omega_{t}=693 \mathrm{meV}$, close to the experimental value. The absorption (dashed line) also exhibits two well-defined peaks but separated by a smaller value $\Omega_{a}=640 \mathrm{meV}$.

Our simulation can be used to study how energy loss distributes among different parts of the system, which is impossible to retrieve by experiment. The (local) dissipation rate is given by $P_{D}(z)=1 / 2 \operatorname{Im}\{\varepsilon\} \omega|E|^{2}$, with $\varepsilon$ the dielectric function and $\omega$ the frequency. Defining $\Pi_{D}$ as the dissipation rate normalized by the incoming energy flux, we get a measure proportional to the number of photons absorbed per unit volume and which is shown in Fig. 3(c). We integrate $\Pi_{D}(z)$, once over the entire cavity length which is equal to $A$ by definition [dashed line in

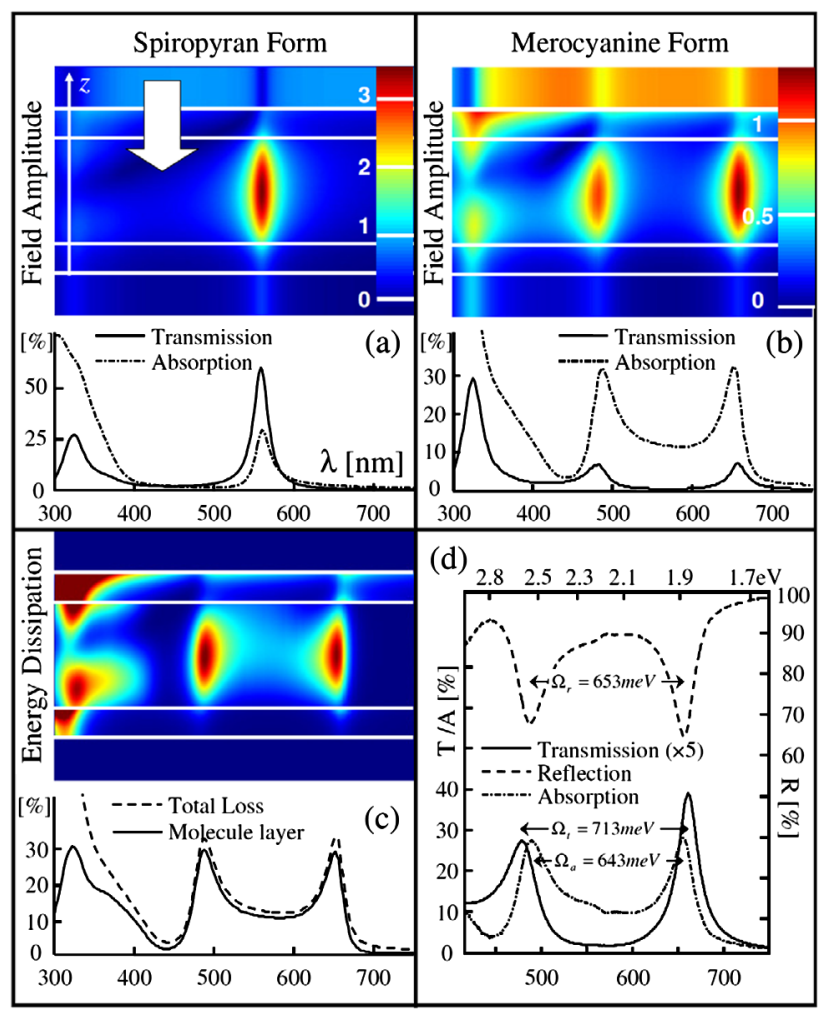

FIG. 3 (color online). Numerical simulations for an SPI (a) and MC (b), (c) cavity. (a) and (b) show the field distribution, with the structure being illuminated from the top. The white lines represent the edges of the Ag layers. The graphs show the transmission intensity (solid line) and total absorption (dash-dotted line). (c) Dissipation rate $\Pi_{D}$ calculated for the MC (strongly coupled) cavity. The graphs show the $z$-direction integrated dissipation rate for the whole structure (dashed line) and the molecule layer alone (solid line). (d) Experimentally measured spectra under SC. The transmission is multiplied by 5 for ease of comparison.
Fig. 3(c)] and once over the molecular layer (solid line). While energy can be seen to be dissipating in the mirrors, this occurs mainly in the UV as expected from the refractive index of $\mathrm{Ag}$. In the visible spectrum where the SC occurs, the energy is dissipated primarily in the molecules, around the new eigenstates. This alteration of the molecules' behavior once placed inside a cavity is a typical manifestation of strong light-matter interaction.

In the context of these results, we go back to the experiment, measure the splitting in reflection $\left(\Omega_{r}\right)$ and absorption, and compare to the transmission data as shown in Fig. 3(d). The splitting is dependent on the measurement type with $\Omega_{t}>\Omega_{r}>\Omega_{a}$ in agreement with theoretical predictions [4]. The observed values as well as the spectral shapes are also in excellent agreement with the simulated ones. For these parameters, the ratio $\Omega_{a} / \Omega_{t}$ is $\sim 0.9$, and we find that it decreases for smaller coupling strengths, leading eventually to a situation where a splitting is observed in transmission but not in absorption. Only a splitting in absorption or emission is an absolute proof of SC [4]. It should be noted that not all the molecules are strongly coupled due to their random distribution and the localized nature of the molecular excitation [24,25]. However, the fraction of strongly coupled molecules increases with coupling strength. In our experiments, this shows up in that the residual absorbance at the original molecular resonance decreases, reaching ca. $20 \%$ at our maximal coupling strength. That is, roughly $80 \%$ of the MC molecules are strongly coupled. Alternatively, this fraction can be estimated from the polariton dispersion [24]. Performing the analysis of Ref. [24] we find that the polaritons retain their coherent nature up to $k_{x}^{\max }=$ $7 \times 10^{8} \mathrm{~m}^{-1}$. With an intermolecular distance $\bar{r}$ of $1 \mathrm{~nm}$, the fraction of coupled states is given by $\left(k_{x}^{\max } \bar{r}\right)^{2}$, which yields $50 \%$.

In the cavity system, the molecules are coupled to the confined EM mode. A different approach to light-matter $\mathrm{SC}$ is via the interaction of molecules with surface plasmons (SPs) [14-16,26]. Like a cavity, SPs provide field confinement and enhancement, which makes them an interesting system for the study of SC, due to their rich behavior and nanoscale integration capabilities. In particular, strong coupling of SPs and molecules was studied in periodically structured metal films (i.e., hole arrays), which offer tunability of SP properties and their interaction with molecules $[15,27]$. By changing the structure period $P$, an SP mode with a particular momentum $k_{\mathrm{SP}}=2 \pi / P$ is excited, appearing as a transmission resonance. A natural question is whether SC is of comparable magnitude in cavities and SP structures for similar conditions. For this purpose a series of hole arrays with different periods were prepared and covered with the PMMA-photochrome film used for the cavity. The transmission spectra of the hole arrays were recorded before and after photoconversion, as shown in Fig. 4. In the SPI form [Fig. 4(a)], the dispersion of the bare SP is apparent. Note that the $Q$ factor of a hole-array resonance is typically $\sim 10$, similar to the cavity. 


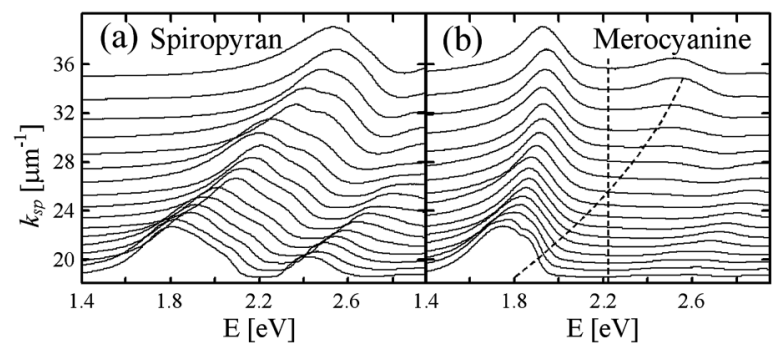

FIG. 4. Dispersion of a hole-array-photochrome system in noncoupled SPI form (a) and MC form (b), where strong coupling is observed as the splitting into two branches. The dispersion was obtained by measuring transmission spectra for a series of hole arrays of different periodicities $(P=180$, $190, \ldots, 340 \mathrm{~nm}$ ), milled on a $200 \mathrm{~nm}$ thick Ag film, with a hole diameter of $P / 2$. The vertical dashed line marks the transition energy of the molecule at the MC form, while the dashed line is a parabolic fit to the bare plasmon mode of (a).

Upon switching to the MC form, the transmission splits into two branches, signifying SC. The high-energy branch is less visible at the crossing point due to Fano-type interference [28]. Nevertheless, the Rabi splitting can be measured to be $650 \mathrm{meV}$, comparable to the value obtained in the cavity. As a consequence, the SP system, being open on one side, provides an interesting alternative to a cavity as it offers easy access for further experiments.

In conclusion, we demonstrated strong coupling of photochromic molecules exhibiting Rabi-splitting values exceeding $700 \mathrm{meV}$ which at $1 / 3$ of the molecular transition energy enters the regime of ultrastrong coupling. In absolute scale, this is to our knowledge the highest Rabi splitting ever observed, and it corresponds to a wavelength of $1.77 \mu \mathrm{m}$, approaching optical frequencies. This value implies coherent light-matter interactions on an extremely short time scale (6 fs), which potentially has implications for various energy transfer and frequency conversion schemes. In the plasmonic version, nanostructuring of the metal can be used to further engineer the light field and thereby the light-matter interactions. With its molecular functionality, our system serve as a tool to explore physical and chemical properties under strong coupling.

The authors acknowledge financial support from the European Research Council (Grant No. 227577).

*ebbesen@unistra.fr

[1] E. M. Purcell, Phys. Rev. 69, 681 (1946); P. Goy et al., Phys. Rev. Lett. 50, 1903 (1983).

[2] Y. Kaluzny et al., Phys. Rev. Lett. 51, 1175 (1983); M. G. Raizen et al., Phys. Rev. Lett. 63, 240 (1989).

[3] A. Boca et al., Phys. Rev. Lett. 93, 233603 (2004).

[4] V. Savona et al., Solid State Commun. 93, 733 (1995); R. Houdré, Phys. Status Solidi B 242, 2167 (2005).

[5] G. Khitrova et al., Nature Phys. 2, 81 (2006).
[6] C. Weisbuch et al., Phys. Rev. Lett. 69, 3314 (1992); J. P. Reithmaier et al., Nature (London) 432, 197 (2004); T. Yoshie et al., Nature (London) 432, 200 (2004).

[7] D. Dini et al., Phys. Rev. Lett. 90, 116401 (2003); A. A. Anappara et al., Appl. Phys. Lett. 91, 231118 (2007).

[8] P. V. Kelkar et al., Phys. Rev. B 56, 7564 (1997); M. Saba et al., Nature (London) 414, 731 (2001).

[9] J. Kasprzak et al., Nature (London) 443, 409 (2006); D. Snoke and P. Littlewood, Phys. Today 63, No. 8, 42 (2010).

[10] J. M. Raimond, M. Brune, and S. Haroche, Rev. Mod. Phys. 73, 565 (2001); T. H. Stievater et al., Phys. Rev. Lett. 87, 133603 (2001).

[11] V.M. Agranovich, H. Benisty, and C. Weisbuch, Solid State Commun. 102, 631 (1997); D. G. Lidzey et al., Nature (London) 395, 53 (1998); D. G. Lidzey et al., Phys. Rev. Lett. 82, 3316 (1999).

[12] R. J. Holmes and S. R. Forrest, Phys. Rev. Lett. 93, 186404 (2004); F. Sasaki, S. Haraichi, and S. Kobayashi, Quantum Electron. 38, 943 (2002); J. R. Tischler et al., Phys. Rev. Lett. 95, 036401 (2005).

[13] P. A. Hobson et al., Appl. Phys. Lett. 81, 3519 (2002).

[14] J. Bellessa et al., Phys. Rev. Lett. 93, 036404 (2004).

[15] J. Dintinger et al., Phys. Rev. B 71, 035424 (2005); A. Salomon, C. Genet, and T. W. Ebbesen, Angew. Chem., Int. Ed. 48, 8748 (2009).

[16] T. K. Hakala et al., Phys. Rev. Lett. 103, 053602 (2009).

[17] C. Ciuti, G. Bastard, and I. Carusotto, Phys. Rev. B 72, 115303 (2005); S. De Liberato, C. Ciuti, and I. Carusotto, Phys. Rev. Lett. 98, 103602 (2007).

[18] A. A. Anappara et al., Phys. Rev. B 79, 201303(R) (2009); Y. Todorov et al., Phys. Rev. Lett. 105, 196402 (2010); T. Niemczyk et al., Nature Phys. 6, 772 (2010).

[19] G. Günter et al., Nature (London) 458, 178 (2009).

[20] G. Berkovic, V. Krongauz, and V. Weiss, Chem. Rev. 100, 1741 (2000); N. Tamai and H. Miyasaka, Chem. Rev. 100, 1875 (2000).

[21] K. Sasaki and T. Nagamura, J. Appl. Phys. 83, 2894 (1998); J. Dintinger, S. Klein, and T. W. Ebbesen, Adv. Mater. 18, 1267 (2006); D. Sridharan et al., Appl. Phys. Lett. 96, 153303 (2010).

[22] A. A. Anappara et al., Superlattices Microstruct. 41, 308 (2007).

[23] Y. Zhu et al., Phys. Rev. Lett. 64, 2499 (1990).

[24] V. M. Agranovich, M. Litinskaia, and D. G. Lidzey, Phys. Rev. B 67, 085311 (2003).

[25] R. Houdré, R. P. Stanley, and M. Ilegems, Phys. Rev. A 53, 2711 (1996).

[26] V. M. Agranovich and A. G. Malshukov, Opt. Commun. 11, 169 (1974); I. Pockrand and A. Brillate, and D. Möbius, J. Chem. Phys. 77, 6289 (1982).

[27] C. Genet and T. W. Ebbesen, Nature (London) 445, 39 (2007).

[28] C. Genet, M.P. van Exter, and J.P. Woerdman, Opt. Commun. 225, 331 (2003). 